\title{
LINEAR INSTABILITY OF SOLITARY WAVE SOLUTIONS OF THE KAWAHARA EQUATION AND ITS GENERALIZATIONS*
}

\author{
THOMAS J. BRIDGES ${ }^{\dagger}$ AND GIANNE DERKS ${ }^{\dagger}$
}

\begin{abstract}
The linear stability problem for solitary wave states of the Kawahara-or fifthorder KdV-type - equation and its generalizations is considered. A new formulation of the stability problem in terms of the symplectic Evans matrix is presented. The formulation is based on a multisymplectification of the Kawahara equation, and leads to a new characterization of the basic solitary wave, including changes in the state at infinity represented by embedding the solitary wave in a multiparameter family. The theory is used to give a rigorous geometric sufficient condition for instability. The theory is abstract and applies to a wide range of solitary wave states. For example, the theory is applied to the families of solitary waves found by Kichenassamy-Olver and Levandosky.
\end{abstract}

Key words. solitary waves, Evans function, multisymplectic structures

AMS subject classifications. $35,53,76$

PII. S0036141099361494

1. Introduction. The Kawahara equation-or fifth-order KdV-type equationis a model equation for plasma waves, capillary-gravity water waves, and other dispersive phenomena when the cubic KdV-type dispersion is weak. Such equations can be written in the general form

$$
2 \frac{\partial u}{\partial t}+\alpha \frac{\partial^{3} u}{\partial x^{3}}+\beta \frac{\partial^{5} u}{\partial x^{5}}=\frac{\partial}{\partial x} f\left(u, u_{x}, u_{x x}\right),
$$

for the scalar-valued function $u(x, t)$, where $\alpha$ and $\beta$ are real parameters with $\beta \neq 0$ and $f\left(u, u_{x}, u_{x x}\right)$ is some smooth function. In many applications the phenomena which lead to the model equation (1.1) have a Hamiltonian structure. Therefore it is natural to require that $f$ be a variational derivative, in which case (1.1) is a Hamiltonian system

$$
\frac{\partial u}{\partial t}=\mathcal{J} \frac{\delta \mathcal{H}}{\delta u}, \quad \text { with } \quad \mathcal{J}=-\frac{1}{2} \frac{\partial}{\partial x},
$$

and

$$
\mathcal{H}(u)=\int_{\mathbb{R}}\left(\frac{1}{2} \beta u_{x x}^{2}-\frac{1}{2} \alpha u_{x}^{2}+h\left(u, u_{x}, u_{x x}\right)\right) d x,
$$

where the variational derivative of the functional associated with $h\left(u, u_{x}, u_{x x}\right)$ yields $f$. Precise forms for $f$ and $h$ will be given in section 2 .

The form of (1.1) which occurs most often in applications is with $f\left(u, u_{x}, u_{x x}\right)=$ $a u^{2}$, where $a$ is a nonzero constant. The first appearance of this equation known to the authors is in the Japanese literature: Kawahara [24] points out that Kakutani and Ono suggested the inclusion of a fifth-order term to KdV to model magnetoacoustic waves in 1969, and Hasimoto first showed in 1970 that a fifth-order term was

${ }^{*}$ Received by the editors September 20, 1999; accepted for publication (in revised form) January 13, 2002; published electronically May 23, 2002.

http://www.siam.org/journals/sima/33-6/36149.html

${ }^{\dagger}$ Department of Mathematics and Statistics, University of Surrey, Guildford, Surrey, GU2 7XH, UK (T.Bridges@surrey.ac.uk, G.Derks@surrey.ac.uk). 
necessary to model capillary-gravity waves for Bond number near one third. Kawahara [24] appears to have been the first to write down the complete equation (1.1) with $f\left(u, u_{x}, u_{x x}\right)=-3 u^{2}$ (see equation (1) in [24]), begin a systematic study, observe that the solitary wave states could have oscillatory tails, and compute examples of such waves numerically. A more general nonlinearity was derived for water waves by Olver [28], using Hamiltonian perturbation theory, with further generalization given by Craig and Groves [13]. Kichenassamy and Olver [25] suggested taking the most reasonable general form for $f$-including nongradient forms - and then deducing under what conditions explicit solitary wave solutions exist, and Levandosky [26] proposed an interesting class of homogeneous nonlinearities. All of the above proposed nonlinearities can be characterized in the form (1.1), and when $f$ is variational the system has the Hamiltonian formulation (1.2).

The system (1.1) has many classes of solutions, but a class of great interest is solitary wave states that are biasymptotic to a constant state at infinity. Depending on the form of the nonlinearity, the system can also have travelling fronts (a simple example is given on page 452 of [15]) as well as solitary waves biasymptotic to invariant manifolds more complex than the lines to be considered here (cf. section 2 and the comments in section 8 of [7]). However, for definiteness, we will restrict attention here to classes of solitary waves which decay exponentially to a constant (in general nonzero) at infinity. Such solitary waves travelling at speed $c$ (i.e., $u(x, t)=\hat{u}(x-c t)$ ) satisfy the fourth-order ordinary differential equation

$$
\beta \hat{u}_{x x x x}+\alpha \hat{u}_{x x}-2 c \hat{u}-f\left(\hat{u}, \hat{u}_{x}, \hat{u}_{x x}\right)=A,
$$

where $A$ is a constant of integration. When $f$ is a gradient operator it is easily shown that (1.4) is the Euler-Lagrange equation associated with a Lagrange functional, and the Legendre transform of this functional results in a Hamiltonian formulation for $(1.4)$,

$$
U_{x}=J \nabla H(U), \quad U \in \mathbb{R}^{4},
$$

where $J$ is a standard unit symplectic operator on $\mathbb{R}^{4}$, and an expression for $H(U)$ is easily deduced but is not needed here. (A more general derivation of such finitedimensional Hamiltonian system will be a consequence of multisymplectic formulation in section 2.)

Note that the Hamiltonian structure of this ODE with $x$ considered as an evolution direction is distinct and dramatically different from the infinite-dimensional Hamiltonian structure associated with the time direction (1.2). The interplay between these two distinct structures will play an important role in what follows.

The Hamiltonian structure (1.5) of the reduced system (1.4) has been the basis of many of the methods for finding solitary wave states. A review article on the known classes of solitary wave states with an exhaustive list of references is given by Champneys [11]. Also of interest in this paper are the class of solitary waves found by Kichenassamy and Olver [25] and the recent results of Levandosky [26]. In [25], a classification of admissible expressions for $f$ which lead to explicit $\operatorname{sech}^{2}$ solitary wave states is given. In [26], an energy-momentum argument is used to prove the existence of a class of solitary waves associated with a homogeneous nonlinearity, and in Groves [19], the mountain-pass lemma is used to prove the existence of solitary waves including multibump solitary waves for a class of homogeneous nonlinearities.

Given the existence of such a large range of solitary wave states for (1.4), a natural question is to determine whether they are stable or unstable. The most successful 
approaches for studying the stability of KdV and generalized KdV (i.e., (1.1) with $\beta=0$ ) have been the energy-momentum method for establishing nonlinear stability and instability (Benjamin [2], Bona [3]) and the connection between the derivative of the momentum with respect to the wave speed and stability (Bona, Souganidis, and Strauss [4], Pego and Weinstein [29]). These energy-momentum based methods have been extended to apply to the stability of solitary waves for the fifth-order KdV by several authors.

The momentum for (1.1) can be expressed as

$$
\mathcal{I}(u)=\int_{\mathbb{R}} u^{2} d x
$$

and therefore solitary wave states can be characterized as solutions of $\delta \mathcal{H}=c \delta \mathcal{I}$, i.e., as critical points of the Hamiltonian restricted to level sets of the momentum with $c$ as a Lagrange multiplier. The nondegeneracy condition for this constrained variational principle is

$$
\frac{d}{d c} \mathcal{I}(\hat{u}) \neq 0
$$

where $\hat{u}(x, c)$ is the family of solitary waves parametrized by $c$. Rigorous Lyapunov stability can be obtained by proving that $\hat{u}$ is indeed a minimizer for this variational principle. This approach has been very successful for KdV-type equations but is very difficult to generalize to higher-order equations and systems of evolutionary PDEs. However, for some range of parameters and forms for $f$, Lyapunov-type energy-momentum arguments have been successfully applied to (1.1). The first results of this type are given by Ill'ichev and Semenov [21] for the waves of depression when $\alpha<0$ which travel at speed $-c$. Karpman [23] shows that when $\alpha=+1$, $\beta<0$, and $f=-\frac{u^{p+1}}{p+1}$ the energy-momentum argument and the sign of $\frac{d I}{d c}$ precisely determine stability and instability. However, this theory relies on a hypothesis that a certain linear operator has exactly one negative eigenvalue which is difficult to verify in general. Karpman's theory is applied by Dey, Khare, and Kumar [15] to a class of exact solutions, but it appears that this class of solutions is explicit only for isolated values of $c$ (see further comments on this at the end of section 3).

Using the energy-momentum method and a compensated compactness argument, Levandosky [26] proves the existence of solitary waves for a homogeneous nonlinearity and obtains rigorous stability and instability results using an energy-momentum argument and the sign of $\frac{d I}{d c}$ for a restricted range of parameter space. Recently, Dias and Kuznetsov [16] have obtained rigorous lower bounds on the Hamiltonian function for (1.1) when $f=-u^{2}$ for the solitary waves with oscillatory tails known to exist near the minimum of the dispersion curve, suggesting that at least one of these families of waves is stable.

For general PDEs (not necessarily Hamiltonian), the most successful approach for the analysis of the linear stability problem is based on the Evans function. The Evans function is a complex analytic function of the spectral parameter, and under suitable hypotheses the zeros of the Evans function correspond to eigenvalues (cf. Evans [17], Alexander, Gardner, and Jones [1]). In Bridges and Derks [7], [9], [10] the concept of the symplectic Evans function and the symplectic Evans matrix were introduced for Hamiltonian evolution equations. This theory, which will be used as a basis for analyzing the linear stability problem for (1.1), will be summarized in sections 2-3. Essentially, the Hamiltonian PDE is reformulated as a Hamiltonian system on a multisymplectic structure, where a distinct symplectic structure is assigned for the time 
and space directions (cf. Bridges [5], [6]). This decomposition allows for a geometric analysis of each step of the existence and linear stability analysis and can be used to deduce an explicit geometric condition for linear instability.

The purpose of this paper is fourfold: first, in section 2, we show that the natural geometric structure of (1.1) is not as a Hamiltonian system as in (1.2) but as a Hamiltonian system on a multisymplectic structure. The problem with (1.2) is that it does not encode any information about the spatial Hamiltonian structure (1.5) that arises when looking for solitary waves and in the linearization about a solitary wave. This geometry should be useful in other analyses of (1.1). Second, in section 3, we show that - with $\alpha, \beta$, and $f$ fixed - all existing solitary wave solutions come in threeparameter families, and these families are a natural consequence of the geometric structure. One of the parameters is $c$, the wave speed, and the other two are related to a space-time drift along an affine group orbit, and when nonzero, they lead to a nontrivial constant state at infinity, and they encode information about the linear stability problem (cf. section 5). We have not found any nontrivial effect on stability of the additional parameters, but we consider only a few examples here. (Examples where nontriviality of the state at infinity affects stability can be found in [7], [9].) These additional parameters are an intrinsic part of the geometry of the PDE. Third, in sections 4-6, we formulate the symplectic Evans matrix for this system. This matrix is of interest because zeros of the determinant of the symplectic Evans matrix in the right-half complex plane correspond to unstable eigenvalues. Fourth, in section 8 , we present a rigorous geometric condition for instability for a class of solitary wave states of (1.1) based on the theory in [9], and then, in sections 9-10, this geometric instability criterion is applied to two examples of families of solitary wave states in the literature.

2. Multisymplectic structure of the Kawahara equation. The starting point for the analysis is the Kawahara equation and its generalizations (1.1), where $f$ is any function which can be written as the variational derivative of a functional

$$
\frac{1}{2} \int_{\mathbb{R}}\left[h\left(u, u_{x}, u_{x x}\right)\right] d x
$$

A straightforward calculation shows that this implies that $h$ has to be of the form

$$
h(q, r, s)=F(q, r)+s E(q, r)
$$

and therefore

$$
f(q, r, s)=F_{q}(q, r)-r F_{q r}(q, r)-s F_{r r}(q, r)+2 s E_{q}(q, r)+s r E_{r q}(q, r)+r^{2} E_{q q}(q, r) .
$$

This expression for $f$ includes all the nonlinearities in variational form for (1.1) encountered in the literature including [12], [23], [24], [25], [26], and [28].

Levandosky [26] considers (1.1) with the restriction that $E=0$ and $F(q, r)$ is three-times continuously differentiable and homogeneous of degree $p+1$ for some $p>1$; that is,

$$
F(\lambda q, \lambda r)=\lambda^{p+1} F(q, r)
$$

for all $\lambda \geq 0$ and $(q, r) \in \mathbb{R}^{2}$.

Kichenasammy and Olver [25] consider the existence of solitary waves for a generalized Kawahara equation, where they assume the existence of a smooth function 
$g(u)$ and constants $A, B$ such that

$$
f(q, r, s)=A r^{2}+B s q+g^{\prime}(q) .
$$

They show that a necessary and sufficient condition for the existence of $\operatorname{sech}^{2}$-type solitary wave solutions is that $g^{\prime}(q)$ be a cubic polynomial. On the other hand, this function $f$ has a variational structure if and only if $2 A=B$, and in this case, the function $f$ can be derived from $h(q, r, s)$ by taking $E=0$ and $F(q, r)=-A q r^{2}+g(q)$.

To reformulate (1.1) with the variational condition (2.1) on $f$, as a Hamiltonian system on a multisymplectic structure, we introduce the potential function $q_{1}(x, t)$, defined by $u=\frac{\partial q_{1}}{\partial x}$. Then with

$$
\begin{aligned}
q_{2}=u=\frac{\partial q_{1}}{\partial x}, & p_{1}=\frac{\partial q_{1}}{\partial t}-\frac{\partial p_{2}}{\partial x}-\frac{\partial}{\partial q_{2}} F-\frac{1}{\beta} E \frac{\partial}{\partial q_{2}} E-\frac{p_{3}}{\beta} \frac{\partial}{\partial q_{2}} E, \\
(2.2) q_{3}=u_{x}=\frac{\partial q_{2}}{\partial x}, & p_{2}=-\alpha q_{3}-\frac{\partial p_{3}}{\partial x}-\frac{\partial}{\partial q_{3}} F-\frac{1}{\beta} E \frac{\partial}{\partial q_{3}} E-\frac{p_{3}}{\beta} \frac{\partial}{\partial q_{3}} E, \\
p_{3} & =\beta \frac{\partial q_{3}}{\partial x}-E,
\end{aligned}
$$

(1.1) reduces to

$$
\frac{\partial q_{2}}{\partial t}+\frac{\partial p_{1}}{\partial x}=0
$$

Combining (2.2) and (2.3), the PDE (1.1) can be written in the form

$$
\mathbf{M} Z_{t}+\mathbf{K} Z_{x}=\nabla S(Z), \quad Z \in \mathbb{R}^{6},
$$

where

$Z=\left(\begin{array}{l}q_{1} \\ q_{2} \\ q_{3} \\ p_{1} \\ p_{2} \\ p_{3}\end{array}\right), \quad \mathbf{M}=\left[\begin{array}{rrrrrr}0 & -1 & 0 & 0 & 0 & 0 \\ 1 & 0 & 0 & 0 & 0 & 0 \\ 0 & 0 & 0 & 0 & 0 & 0 \\ 0 & 0 & 0 & 0 & 0 & 0 \\ 0 & 0 & 0 & 0 & 0 & 0 \\ 0 & 0 & 0 & 0 & 0 & 0\end{array}\right], \quad \mathbf{K}=\left[\begin{array}{rrrrrr}0 & 0 & 0 & -1 & 0 & 0 \\ 0 & 0 & 0 & 0 & -1 & 0 \\ 0 & 0 & 0 & 0 & 0 & -1 \\ 1 & 0 & 0 & 0 & 0 & 0 \\ 0 & 1 & 0 & 0 & 0 & 0 \\ 0 & 0 & 1 & 0 & 0 & 0\end{array}\right]$,

and

$$
S(Z)=\frac{1}{2} \alpha q_{3}^{2}+\frac{1}{2 \beta} p_{3}^{2}+p_{1} q_{2}+p_{2} q_{3}+F\left(q_{2}, q_{3}\right)+\frac{1}{2 \beta}\left(2 p_{3}+E\left(q_{2}, q_{3}\right)\right) E\left(q_{2}, q_{3}\right)
$$

The skew-symmetric operators $\mathbf{M}$ and $\mathbf{K}$ define the two-forms

$$
\begin{aligned}
\omega & =\mathbf{d} q_{2} \wedge \mathbf{d} q_{1}, \\
\kappa & =\mathbf{d} p_{1} \wedge \mathbf{d} q_{1}+\mathbf{d} p_{2} \wedge \mathbf{d} q_{2}+\mathbf{d} p_{3} \wedge \mathbf{d} q_{3},
\end{aligned}
$$

with

$$
\omega\left(\xi_{1}, \xi_{2}\right)=\left\langle\mathbf{M} \xi_{1}, \xi_{2}\right\rangle \text { and } \kappa\left(\xi_{1}, \xi_{2}\right)=\left\langle\mathbf{K} \xi_{1}, \xi_{2}\right\rangle,
$$

where $\langle\cdot, \cdot\rangle$ is a standard inner product on $\mathbb{R}^{6}$. The induced norm is denoted by $\|\cdot\|$. The symplectic form $\kappa$ is a canonical symplectic structure on $\mathbb{R}^{6}$ associated with the $x$-direction, and $\omega$ is a rank 2 symplectic structure associated with the $t$-direction. 
There are two symmetries of (2.4) which will be of interest in what follows: the spatial translation invariance (in $x$ ) of the system (i.e., the fact that $\omega, \kappa$, and $S(Z)$ do not depend explicitly on $x$ ), and the affine symmetry associated with the fact that $q_{1}$ is a potential function.

Let $G$ be the one-parameter affine group associated with this potential symmetry with action

$$
\mathcal{G}_{\theta} Z=Z+\theta V \quad \text { for all } \theta \in \mathbb{R}, \quad \text { where } \quad V=\left(\begin{array}{c}
1 \\
0 \\
\vdots \\
0
\end{array}\right) \text {. }
$$

Then the system (2.4) is $G$-equivariant; that is, $S(Z)$ and the two-forms $\omega$ and $\kappa$ are $G$-invariant.

A solitary wave state of (2.4) will be composed of two parts. The first part is the shape of the solitary wave which connects the asymptotic states at plus and minus infinity, which will be characterized as a heteroclinic orbit in the phase space $\mathbb{R}^{6}$. The second part is the state at infinity which will be characterized as an invariant manifold of relative equilibria associated with the group $G$.

To define the invariant manifold at infinity we use the theory in section 2 of [9]. First note that, with $P(Z)=q_{2}$ and $Q(Z)=p_{1}$, the functions $P(Z)$ and $Q(Z)$ satisfy

$$
\mathbf{M} V=\nabla P(Z) \quad \text { and } \quad \mathbf{K} V=\nabla Q(Z) ;
$$

that is, $P(Z)$, respectively, $Q(Z)$, are the functions which generate the $\omega$-, respectively, $\kappa$-, symplectic flow of the group $G$. The state at infinity is taken to be of the form

$$
Z(x, t)=\mathcal{G}_{\theta(x, t)} Z_{0}(a, b) \text { with } \theta(x, t)=a t+b x+\theta_{0} .
$$

The point $Z_{0} \in \mathbb{R}^{6}$ and the parameters $a$ and $b$ are defined by the constrained variational problem: find critical points of $S(Z)$ restricted to level sets of the functions $P$ and $Q$, or

$$
\nabla S\left(Z_{0}\right)=a \nabla P\left(Z_{0}\right)+b \nabla Q\left(Z_{0}\right), \quad \text { with } \quad P\left(Z_{0}\right)=\mathcal{P}, Q\left(Z_{0}\right)=\mathcal{Q} .
$$

This equation is easily solved to find

$$
Z_{0}=\left(\begin{array}{c}
q_{1}^{0} \\
b \\
0 \\
a-F_{q}(b, 0) \\
-F_{r}(b, 0) \\
-E(b, 0)
\end{array}\right) \quad \text { with } \begin{aligned}
& P\left(Z_{0}\right)=q_{2}^{0}=b=\mathcal{P} \\
& Q\left(Z_{0}\right)=p_{1}^{0}=a-F_{q}(b, 0)=\mathcal{Q}
\end{aligned}
$$

and $q_{1}^{0}$ is arbitrary (due to the group action). This state is nondegenerate as a solution of the constrained variational problem since $\frac{\partial(P, Q)}{\partial(a, b)}=-1 \neq 0$.

Let $Z_{0} \in \mathbb{R}^{6}$ be any nondegenerate solution of (2.12) with $q_{1}^{0}=0$. Then the invariant manifold at infinity is defined to be the following line in $\mathbb{R}^{6}$ through $Z_{0}$ :

$$
\mathcal{M}_{\infty}=\left\{Z_{0}+\theta V: \theta \in \mathbb{R}\right\} .
$$

The solitary wave state will be taken to be biasymptotic to this manifold and of the form

$$
Z(x, t)=\mathcal{G}_{\theta(x, t)}\left[Z_{0}^{-}+\mathcal{T}_{\tau(t)} \widehat{Z}(x, a, b, c)\right]
$$


where $\mathcal{G}_{\theta(x, t)}$ is as defined in (2.11); $Z_{0}^{-}$is any nondegenerate solution of (2.12) with $q_{1}^{0}=0$ (the "-" superscript indicates that this is the asymptotic point on $\mathcal{M}_{\infty}$ as $x \rightarrow-\infty)$;

$$
\mathcal{T}_{\tau} \widehat{Z}(x, a, b, c) \stackrel{\text { def }}{=} \widehat{Z}(x-\tau, a, b, c),
$$

and $\tau(t)=c t+\tau_{o}$. The function $\widehat{Z}(x, a, b, c)$, which is the shape of the solitary wave, is a heteroclinic orbit of the Hamiltonian system on $\mathbb{R}^{6}$,

$$
\mathbf{J}_{c} \widehat{Z}_{x}=\nabla W(\widehat{Z}), \quad \widehat{Z} \in \mathbb{R}^{6}
$$

with

$$
\mathbf{J}_{c}=\mathbf{K}-c \mathbf{M}, \quad W(\widehat{Z})=S\left(Z_{0}^{-}+\widehat{Z}\right)-a P\left(Z_{0}^{-}+\widehat{Z}\right)-b Q\left(Z_{0}^{-}+\widehat{Z}\right) .
$$

The symplectic operator $\mathbf{J}_{c}$ is nondegenerate and defines the symplectic structure $\left(\mathbb{R}^{6}, \Omega\right)$, where $\Omega=\kappa-c \omega$.

This Hamiltonian system is the analogue of the Hamiltonian ODE presented in (1.5). There are, however, two important differences: the symplectic structure $\Omega$ is defined explicitly in terms of a combination of the spatial $(\kappa)$ and temporal $(\omega)$ structures, and $c$ appears here explicitly as a multiplier of the temporal symplectic structure $\omega$. In other words, even though (1.5) is Hamiltonian there is no connection with the spatial or temporal symplectic structure of the full system (1.1), while (2.16) still contains these connections.

The heteroclinic orbit $\widehat{Z}(x, a, b, c)$ satisfies the asymptotic conditions

$$
\lim _{x \rightarrow-\infty}\|\widehat{Z}(x, a, b, c)\|=0 \quad \text { and } \quad \lim _{x \rightarrow \infty}\left\|\widehat{Z}(x, a, b, c)-Z_{0}^{+}+Z_{0}^{-}\right\|=0,
$$

where $Z_{0}^{+}=\mathcal{G}_{\gamma} Z_{0}^{-}$for some $\gamma \in G$. In other words, as $x \rightarrow+\infty$ the function $\widehat{Z}(x, a, b, c)$ is asymptotic to a point on $\mathcal{M}_{\infty}$ other than $Z_{0}^{-}$, but this point is related to $Z_{0}^{-}$by an element $\gamma$ in the group $G$. In the present case, the difference in $Z_{0}^{+}$and $Z_{0}^{-}$corresponds to a jump in the value of the potential $q_{1}$.

3. A three-parameter family of solitary waves. For the linearized stability theory, we will assume the existence of open sets $A, B$, and $C$ in $\mathbb{R}$ such that for each $(a, b, c) \in A \times B \times C$ there exists a bounded travelling wave shape $\widetilde{Z}(x ; a, b, c)=$ $Z_{0}^{-}(a, b, c)+\widehat{Z}(x ; a, b, c)$, which satisfies

$$
\mathbf{J}_{c} \widetilde{Z}_{x}=\nabla S(\widetilde{Z})-a \nabla P(\widetilde{Z})-b \nabla Q(\widetilde{Z}) .
$$

Furthermore, we assume that the derivative of the shape of the solitary wave, $\widetilde{Z}_{x}$, is exponentially decaying with asymptotic estimate

$$
\lim _{x \rightarrow \pm \infty} e^{ \pm \delta x} \widetilde{Z}_{x}=\Psi^{ \pm} \text {and } \lim _{x \rightarrow \pm \infty} \partial_{x}\left[e^{ \pm \delta x} \widetilde{Z}_{x}\right]=0
$$

for some $\Psi^{ \pm} \in \mathbb{R}^{6}$ and $\delta>0$. This assumption is in general easy to verify for solitary waves which are explicitly known. Indeed the above two hypotheses are very unrestrictive and cover a wide range of known solitary waves.

The bounded travelling wave shapes $\widetilde{Z}$ will be asymptotic to the points $Z_{0}^{+}$and $Z_{0}^{-}$for $x \rightarrow \infty$, respectively, $x \rightarrow-\infty$. The phase shift between the point on $\mathcal{M}_{\infty}$ at plus and minus infinity are related by using the group action of $G$ : explicitly we find

$$
Z_{0}^{+}=\mathcal{G}_{\gamma} Z_{0}^{-}, \quad \text { where } \quad \gamma=\int_{-\infty}^{\infty}(u(x)-b) d x
$$


and $u(x)=\widetilde{q}_{2}(x)$ is the second component of the solitary wave solution. By the hypotheses, this integral exists and is nonzero in general. It is a generalization of the "mass" of the solitary wave (cf. Longuet-Higgins [27]).

The momentum of the shape of the solitary wave is defined by

$$
I(\widetilde{Z})=\frac{1}{2} \int_{-\infty}^{+\infty} \omega\left(\widetilde{Z}, \widetilde{Z}_{x}\right) d x
$$

where the dependence on $a$ and $b$ has been suppressed. By taking

$$
H(\widetilde{Z})=\int_{-\infty}^{+\infty}\left(\frac{1}{2} \kappa\left(\widetilde{Z}, \widetilde{Z}_{x}\right)+W(\widetilde{Z})\right) d x
$$

it is straightforward to verify that the energy-momentum characterization of solitary waves is encoded in (2.16) and (3.1) (cf. [7], [9]), but this characterization will not be needed explicitly in what follows.

With the above hypotheses, it is shown in [9] that the derivative of $I$ with respect to $c$ exists and takes the form

$$
\frac{d}{d c} I(\widetilde{Z})=\int_{-\infty}^{+\infty} \omega\left(\widetilde{Z}_{c}, \widetilde{Z}_{x}\right) d x+\frac{1}{2} \omega\left(Z_{0}^{+}, \partial_{c} Z_{0}^{+}\right) .
$$

An essential point to note in the interpretation of this expression is that the derivative with respect to $c$ is taken with all other parameters fixed. While this may appear to be obvious, it is easy to be misled into thinking that a family of solutions depends on $c$ when in fact it exists only for a single value of $c$. Examples of this are the explicit solutions found in [12], [14], [15], and [20] which for fixed values of the parameters in the equation exist for a single value of $c$ and therefore $\frac{d I}{d c}$ cannot be explicitly computed. On the other hand, when a solitary wave state is known at an isolated value of $c$, it is not difficult to prove that it persists for a range of $c$ values by a multisymplectic Melnikov argument. In other words, families in $c$ generically exist, even when an explicit solution exists for a single value of $c$ only. An example of the numerical continuation of such an isolated explicit solution can be found in [12].

4. The linearization about a family of solitary waves. To study the stability of a solitary wave $\mathcal{G}_{\theta(x, t)}\left[Z_{0}^{-}+\mathcal{T}_{\tau(t)} \widehat{Z}(x ; a, b, c)\right]$ (see $(2.15)$ ), write $Z(x, t)=$ $\mathcal{G}_{\theta(x)}\left[Z_{0}^{-}+\mathcal{T}_{\tau(t)}[\widehat{Z}(x ; a, b, c)+\widehat{U}(x, t)]\right]$. Then the linearization of $(2.4)$ about the family of solitary waves takes the form

$$
\mathbf{M} \widehat{U}_{t}+\mathbf{J}_{c} \widehat{U}_{x}=\mathbf{B}(x ; a, b, c) \widehat{U},
$$

where

$$
\begin{aligned}
\mathbf{B}(x ; \cdot) & =D^{2} W(\widehat{Z}(x ; \cdot)) \\
& =D^{2} S\left(Z_{0}^{-}+\widehat{Z}(x ; \cdot)\right)-a D^{2} P\left(Z_{0}^{-}+\widehat{Z}(x ; \cdot)\right)-b D^{2} Q\left(Z_{0}^{-}+\widehat{Z}(x ; \cdot)\right)
\end{aligned}
$$

(cf. section 3 of [9]). With the spectral ansatz $\widehat{U}(x, t)=e^{\lambda t} U(x, \lambda)$, the system (4.1) reduces to

$$
U_{x}=\mathbf{A}(x, \lambda) U, \quad U \in \mathbb{C}^{6},
$$

with

$$
\mathbf{A}(x, \lambda)=\mathbf{J}_{c}^{-1}[\mathbf{B}(x ; \cdot)-\lambda \mathbf{M}] .
$$


The dependence on the parameters $(a, b, c)$ in the argument of $\mathbf{A}$ is suppressed, as they are considered fixed in the stability analysis. The matrix $\mathbf{A}(x, \lambda)$ has the following asymptotic limits:

$$
\lim _{x \rightarrow \pm \infty} \mathbf{A}^{\infty}(\lambda)=\mathbf{J}_{c}^{-1}\left[\mathbf{B}^{\infty}-\lambda \mathbf{M}\right]
$$

where

$$
\mathbf{B}^{\infty}=\lim _{x \rightarrow \pm \infty} \mathbf{B}(x ; \cdot)=D^{2} S\left(Z_{0}\right)-a D^{2} P\left(Z_{0}\right)-b D^{2} Q\left(Z_{0}\right),
$$

with $Z_{0}$ either $Z_{0}^{-}$or $Z_{0}^{+}$. It is a consequence of the results in [9] that although $Z_{0}^{-} \neq Z_{0}^{+}$, the linearization $\mathbf{B}^{\infty}$ will be the same at $\pm \infty$. Explicitly, $\mathbf{B}^{\infty}$ is

$$
\mathbf{B}^{\infty}=\left(\begin{array}{cccccc}
0 & 0 & 0 & 0 & 0 & 0 \\
0 & F_{q q}(b, 0)+\frac{E_{q}(b, 0)^{2}}{\beta} & F_{q r}(b, 0)+\frac{E_{q}(b, 0) E_{r}(b, 0)}{\beta} & 1 & 0 & \frac{E_{q}(b, 0)}{\beta} \\
0 & F_{q r}(b, 0)+\frac{E_{q}(b, 0) E_{r}(b, 0)}{\beta} & \alpha+F_{r r}(b, 0)+\frac{E_{r}(b, 0)^{2}}{\beta} & 0 & 1 & \frac{E_{r}(b, 0)}{\beta} \\
0 & 1 & 0 & 0 & 0 & 0 \\
0 & 0 & 1 & 0 & 0 & 0 \\
0 & \frac{E_{q}(b, 0)}{\beta} & \frac{E_{r}(b, 0)}{\beta} & 0 & 0 & \frac{1}{\beta}
\end{array}\right) .
$$

The spectrum of $\mathbf{A}^{\infty}(\lambda)$ is defined by

$$
\sigma\left(\mathbf{A}^{\infty}(\lambda)\right)=\{\mu \in \mathbb{C}: \Delta(\mu, \lambda)=0\}
$$

where

$$
\Delta(\mu, \lambda)=\operatorname{det}\left[\mathbf{B}^{\infty}-\mu \mathbf{J}_{c}-\lambda \mathbf{M}\right], \quad \lambda \in \Lambda .
$$

The set $\Lambda \in \mathbb{C}$ is some subset of the right-half complex $\lambda$-plane, which will be identified later. A straightforward calculation shows that

$$
\Delta(\mu, \lambda)=\mu^{6}+\frac{C_{1}}{\beta} \mu^{4}-\frac{C_{2}+2 c}{\beta} \mu^{2}+2 \frac{\lambda}{\beta} \mu,
$$

where $C_{1}=F_{r r}(b, 0)-2 E_{q}(b, 0)+\alpha$ and $C_{2}=F_{q q}(b, 0)$.

This expression shows that $\mu=0$ is an eigenvalue for the linearized system for any value of $\lambda$. The solution of the linearized equation related to this eigenvalue is independent of $x$ and is given explicitly by

$$
U=(1,0,0, \lambda, 0,0) .
$$

This zero eigenvalue and its eigenvector are reminiscent of a similar phenomenon that appears with $\mathrm{KdV}$; see section 6 of [7]. It arises due to the introduction of a potential for $u(x, t)$.

We can also determine the eigenvectors associated with each of the other five $\mu$-eigenvalues; they are

$$
U_{\mathrm{ev}}(\mu, \lambda)=\left(1, \mu, \mu^{2},-\lambda+\mu c,-\mu F_{1},-\mu F_{2}\right), \quad \mu \neq 0,
$$

where

$$
F_{1}=F_{q r}(b, 0)+\mu\left(C_{1}+E_{q}(b, 0)\right)+\beta \mu^{3} \quad \text { and } \quad F_{2}=E_{q}(b, 0)+\mu E_{r}(b, 0)-\beta \mu^{2} .
$$

If $\mu \in i \mathbb{R} \backslash\{0\}$, then $\lambda \in i \mathbb{R}$. Therefore, if $\operatorname{Re}(\lambda)>0$, the only solution in $\sigma\left(\mathbf{A}^{\infty}(\lambda)\right) \cap i \mathbb{R}$ is the trivial state (4.4). 


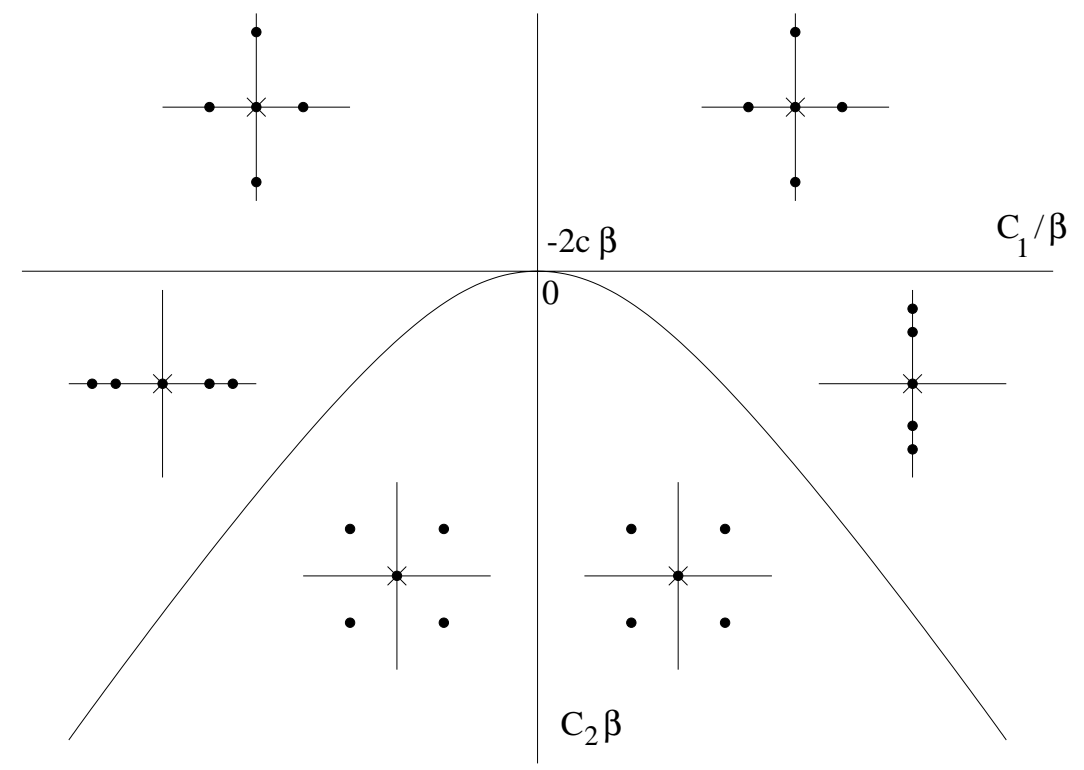

FIG. 4.1. Sketch of the position of the eigenvalues $\mu$ at $\lambda=0$ as a function of $C_{1} / \beta$ and $C_{2} \beta$. The parabolic curve represents the relation $C_{1}^{2}=-4 \beta\left(C_{2}+2 c\right)$.

4.1. The linearized equation with $\boldsymbol{\lambda}=\mathbf{0}$. First consider the spectrum of $\mathbf{A}^{\infty}(0)$ which is associated with the existing solitary wave

$$
\Delta(\mu, 0)=\mu^{2}\left(\mu^{4}+\frac{C_{1}}{\beta} \mu^{2}-\frac{C_{2}+2 c}{\beta}\right) .
$$

We can immediately see that at $\lambda=0$, the $\mu$-spectrum is given by

$$
\left\{0,0, \sqrt{-\frac{C_{1}}{2 \beta} \pm \frac{1}{2 \beta} \sqrt{C_{1}^{2}+4 \beta\left(C_{2}+2 c\right)}},-\sqrt{-\frac{C_{1}}{2 \beta} \pm \frac{1}{2 \beta} \sqrt{C_{1}^{2}+4 \beta\left(C_{2}+2 c\right)}}\right\}
$$

A sketch of the position of the eigenvalues $\mu$ as function of $C_{1} / \beta$ and $C_{2} \beta$ is given in Figure 4.1. In order to satisfy the exponential decay condition (3.2) on the solitary wave, it is necessary for the spectrum $\Delta(\mu, 0)$ to have at least one pair of strictly hyperbolic eigenvalues. The region with $C_{1} / \beta<0$ and $C_{2} \beta<-2 c \beta$ with four real hyperbolic eigenvalues is the region studied by Karpman [23] using the energymomentum method to prove stability and instability (for the case in (1.1) when $f$ is a polynomial in $u$ ). The region with $C_{1} / \beta>0$ in the neighborhood of the parabola $C_{1}^{2}+4 \beta\left(C_{2}+2 c\right)=0$ is the region studied by Dias and Kuznetsov [16], and in this region they show that the energy-momentum method leads to the existence of a minimum (for the case in (1.1) when $f$ is a quadratic function of $u$ ).

Here we will consider the case where the spectrum of $\mathbf{A}^{\infty}(0)$ has exactly one pair of hyperbolic real eigenvalues and one pair of purely imaginary eigenvalues. In Figure 4.1, this corresponds to the region

$$
C_{2} \beta>-2 c \beta,
$$

and we will concentrate on this part of parameter space. Note that the zero eigenvalue of $\mathbf{A}^{\infty}(0)$ has algebraic multiplicity two and geometric multiplicity one and the 


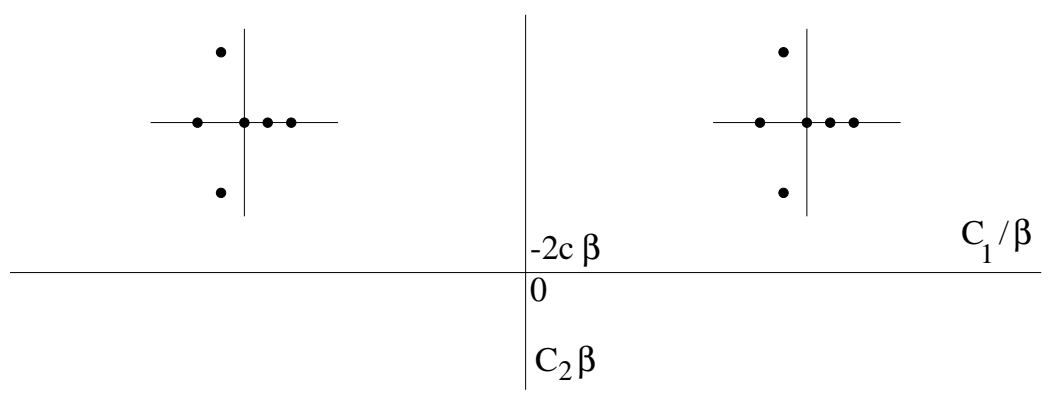

FIG. 4.2. Position of the eigenvalues for $\lambda$ small and $\beta>0$.

eigenvector, $(1,0,0,0,0,0)$, is in fact the generator of the group action of $G$, defined in section 2.

By differentiating (3.1) with respect to $x$, we see that $\widetilde{Z}_{x}$ is a solution of the linearized equation (4.2) with $\lambda=0$. By assumption the derivative of the solitary wave shape $\widetilde{Z}_{x}$ is exponentially decaying, hence both real eigenvalues can be related to the exponential decay rate of the derivative of the solitary wave shape. The negative real eigenvalue is equal to $-\delta$ and the positive real eigenvalue is equal to $\delta$ (see (3.2)), with $\Psi^{ \pm}$the eigenvectors of the systems at $\pm \infty$.

4.2. The linearized equation with $\Re(\lambda)>0$ and $\lambda$ small. Next we consider the linearized equation (4.2) with $\Re(\lambda)>0$ and $\lambda$ small. When $\lambda$ is small, the eigenvalues which were on the imaginary axis when $\lambda=0$ have expansions for $\lambda$ small given by

$$
\begin{aligned}
\mu & =0 \\
\mu & =\frac{2}{C_{2}+2 c} \lambda+\mathcal{O}\left(\lambda^{2}\right) \\
\mu & = \pm \frac{i}{2} \sqrt{2} \sqrt{C_{3}}-\frac{1}{2 \beta} C_{3}\left(C_{3}-\frac{C_{1}}{\beta}\right) \lambda+\mathcal{O}\left(\lambda^{2}\right),
\end{aligned}
$$

where $C_{3}=\frac{C_{1}}{\beta}+\sqrt{\frac{C_{1}^{2}}{\beta^{2}}+\frac{4\left(C_{2}+2 c\right)}{\beta}}$. Since $\beta\left(C_{2}+2 c\right)>0$, the term $-\frac{1}{2 \beta} C_{3}\left(C_{3}-\frac{C_{1}}{\beta}\right)$ has sign opposite to that of $\frac{2}{C_{2}+2 c}$. Hence, if $\beta>0$, the nonzero eigenvalues on the imaginary axis are perturbed to the left and one of the zero eigenvalues is perturbed to the right when $\lambda \neq 0$. The position of the eigenvalues is sketched in Figure 4.2 with $\beta>0$. If $\beta<0$, the movement of the eigenvalues will be in the opposite direction. Hence we have a 4-2 split in the eigenvalues. This means that for $\Re(\lambda)>0$, if $\beta<0$, there are two eigenvalues with negative real part and four eigenvalues with nonnegative real part. And if $\beta>0$, there are two eigenvalues with positive real part and four eigenvalues with nonpositive real part.

A straightforward calculation shows that double eigenvalues, i.e., values of $\lambda$ where

$$
\Delta(\mu, \lambda)=\frac{\partial}{\partial \mu} \Delta(\mu, \lambda)=0,
$$

occur at isolated real values of $\lambda$, explicitly, when

$$
\lambda_{0}= \pm \frac{\beta}{50} \sqrt{\frac{1}{10 \beta}\left(C_{4} \beta-3 C_{1}\right)}\left(\left(C_{4}-\frac{C_{1}}{2 \beta}\right)^{2}-\frac{25 C_{1}^{2}}{4 \beta^{2}}\right),
$$




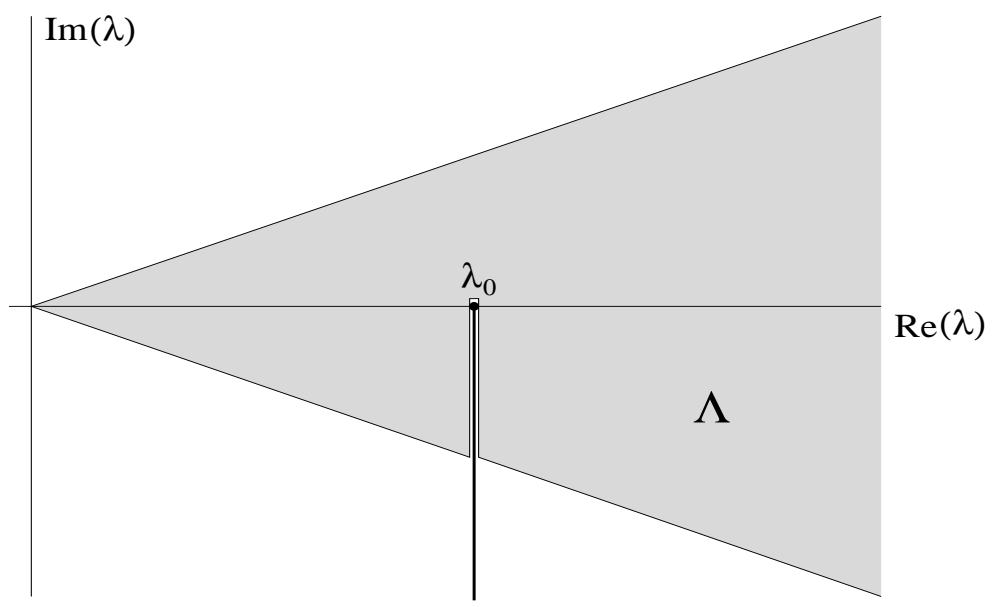

FIG. 4.3. The set $\Lambda_{b}$ in the right-half complex plane in which the eigenvalues are analytic and its extension is $\Lambda$.

where $C_{4}=\sqrt{\frac{9 C_{1}^{2}}{\beta^{2}}+\frac{20\left(C_{2}+2 c\right)}{\beta}}$. A consequence of the condition (4.7) is that $\lambda_{0}$ is always real. The double eigenvalues associated with $\lambda=\lambda_{0}$ are $\mu_{d}= \pm \sqrt{\frac{1}{10 \beta}\left(C_{4} \beta-3 C_{1}\right)}$. If we define

$$
\Lambda_{b}=\{\lambda \in \mathbb{C}|\Re(\lambda)>0,| \Im(\lambda) \mid<\Re(\lambda)\} \backslash\left\{\left|\lambda_{0}\right|+i y \mid y \leq 0\right\},
$$

then the eigenvalues are simple when $\lambda \in \Lambda_{b}$. The region $\Lambda_{b}$ is shown in Figure 4.3.

The set $\Lambda$ will be defined as the extension of the set $\Lambda_{b}$ obtained by removing the branch point and the branch cut.

5. Intermezzo: Temporal drift along the group $G$. In section 2 , the state at infinity associated with the basic solitary wave is $x$ and $t$ dependent and of the form

$$
Z(x, t)=\mathcal{G}_{\theta(x, t)} Z_{0}(a, b)=Z_{0}(a, b)+\theta(x, t) V .
$$

Since only the first component of $V$ is nonzero, the only component of $Z(x, t)$ in this expression which will depend on $x$ and $t$ is the first component. In the multisymplectic coordinates, the first coordinate is $q_{1}(x, t)$, where

$$
u(x, t)=q_{2}(x, t)=\frac{\partial}{\partial x} q_{1}(x, t),
$$

and so

$$
q_{1}(x, t)=q_{1}^{o}(a, b)+\theta(x, t)=a t+b x+\theta_{0} .
$$

Since $q_{1}(x, t)$ is a potential, the $t$-dependence of $q_{1}(x, t)$ will have no dynamic significance for $u(x, t)$ which is the primary function associated with (1.1). It is tempting to conclude that the temporal part of the flow on $G$-represented by the parameter $a$-is irrelevant. Surprisingly, it is not. By embedding the solitary wave in the three-parameter family $(a, b, c)$, rather than the two-parameter family $(b, c)$ (or even 
the one-parameter $(c)$ family), geometric information about the linear stability is obtained, even if we set $a=0$ at the end of the analysis. Here we will indicate two examples of how $a$ encodes geometric information.

The basepoint, $Z_{0}(a, b)$, of the two-parameter family of states at infinity satisfies a constrained variational principle (cf. (2.12) and (2.13)) with the nondegeneracy condition

$$
\operatorname{det}\left(\begin{array}{ll}
\frac{\partial P}{\partial a} & \frac{\partial P}{\partial b} \\
\frac{\partial Q}{\partial a} & \frac{\partial Q}{\partial b}
\end{array}\right) \neq 0
$$

and for the Kawahara family at infinity it was found that

$$
\left(\begin{array}{ll}
\frac{\partial P}{\partial a} & \frac{\partial P}{\partial b} \\
\frac{\partial Q}{\partial a} & \frac{\partial Q}{\partial b}
\end{array}\right)=\left(\begin{array}{cc}
0 & 1 \\
1 & -F_{q q}(b, 0)
\end{array}\right)
$$

and hence the nondegeneracy condition is satisfied for any $a$ and $b$. This information was not used explicitly in the later analysis, but it does appear implicitly in the following way.

In section 4 , the spectral problem associated with the linearization about the solitary wave in the limit as $x \rightarrow \pm \infty$ was associated with the function

$$
\Delta(\mu, \lambda)=\operatorname{det}\left[\mathbf{B}^{\infty}-\mu \mathbf{J}_{c}-\lambda \mathbf{M}\right], \quad \lambda \in \Lambda .
$$

In [9] the following remarkable result is proved for any relative equilibrium at infinity of the abstract form (5.1) (see Lemma 7 in [9]):

$$
\Delta(\mu, \lambda)=\mathbb{C}\left[\frac{\partial Q}{\partial b} \mu^{2}+\left(\frac{\partial Q}{\partial a}+\frac{\partial P}{\partial b}\right) \mu(\lambda-c \mu)+\frac{\partial P}{\partial a}(\lambda-c \mu)^{2}\right]+o\left((|\lambda|+|\mu|)^{2}\right),
$$

where $\mathbb{C}$ represent a nonzero constant. In other words, the perturbation of the $\mu$-roots for $|\lambda|$ small is dictated by the parameter structure encoded in the state at infinity. Substituting the Kawahara expressions into this expansion results in

$$
\Delta(\mu, \lambda)=\mathbb{C}\left[-C_{2} \mu^{2}+2 \mu(\lambda-c \mu)\right]+o\left((|\lambda|+|\mu|)^{2}\right),
$$

using $C_{2}=F_{q q}(b, 0)$. It is evident from this expression that for $|\lambda|$ small the two roots are $\mu=0$ and

$$
\mu=\frac{2}{2 c+C_{2}} \lambda
$$

For $2 c+C_{2}>0$ this result recovers precisely the perturbation result in Figure 4.2. Moreover, it gives a precise geometric description of how the zero $\mu$-roots are perturbed when $\lambda$ is perturbed away from zero for the other regions in Figure 4.1, and this information is an essential part of the construction of the Evans matrix.

A second example where the parameter $a$ has implications is when deducing a geometric instability criterion. Using the symplectic Evans matrix, we will prove a geometric instability condition for solitary waves, based on the theory in [9], where the proof uses in an essential way the parameter structure of the state at infinity, even when only the case $a=b=0$ is of interest. 
6. The symplectic Evans matrix. The system (4.2) with the spectrum of $\mathbf{A}^{\infty}(\lambda)$ as shown in Figure 4.2 is in the appropriate form for construction of the Evans function. The Evans function is constructed as follows (cf. Alexander, Gardner, and Jones [1]).

For fixed $(a, b, c) \in A \times B \times C$ let $\mathbf{U}^{-}(x, \lambda) \in \bigwedge^{2}\left(\mathbb{C}^{6}\right)$, and let $\mathbf{U}^{+}(x, \lambda) \in$ $\Lambda^{4}\left(\mathbb{C}^{6}\right)$. Let $\alpha_{-}(\lambda)$ be the sum of the eigenvalues of $\mathbf{A}^{\infty}(\lambda)$ with positive real part, and let $\alpha_{+}(\lambda)=\tau_{\infty}(\lambda)-\alpha_{-}(\lambda)$, where $\tau(x, \lambda)=\operatorname{Trace}(\mathbf{A}(x, \lambda))$ and $\tau_{\infty}(\lambda)=$ $\lim _{|x| \rightarrow \infty} \tau(x, \lambda)$. Then $\mathbf{U}^{-}(x, \lambda), \mathbf{U}^{+}(x, \lambda)$ are chosen to satisfy induced equations on $\bigwedge^{2}\left(\mathbb{C}^{6}\right)$ and $\bigwedge^{4}\left(\mathbb{C}^{6}\right)$, respectively, and

$$
\lim _{x \rightarrow \pm \infty} \mathrm{e}^{-\alpha_{ \pm}(\lambda) x} \mathbf{U}^{ \pm}(x, \lambda)=\zeta_{ \pm}(\lambda),
$$

where $\zeta_{-}(\lambda)$ and $\zeta_{+}(\lambda)$ are eigenvectors of $\bigwedge^{2}\left(\mathbf{A}_{\infty}(\lambda)\right)$ and $\bigwedge^{4}\left(\mathbf{A}_{\infty}(\lambda)\right)$, respectively, corresponding to the eigenvalues $\alpha_{-}(\lambda)$ and $\alpha_{+}(\lambda)$. The Evans function then takes the form

$$
D(\lambda)=\mathrm{e}^{-\int_{0}^{x} \tau(s, \lambda) \mathrm{d} s} \mathbf{U}^{+}(x, \lambda) \wedge \mathbf{U}^{-}(x, \lambda) \quad \text { for all } \lambda \in \Lambda .
$$

It is independent of $x$ and analytic for all $\lambda \in \Lambda$ [1]. Indeed it is analytic on a larger subset of $\mathbb{C}$, but this extension will not be needed here.

One of the shortcomings of the form (6.1) is that it does not encode in any obvious way the multisymplectic structure of the system (4.2). However, by using individual solutions of (4.2), the symplectic structure can be made explicit.

For fixed $(a, b, c) \in A \times B \times C$ let $U_{i}^{-}(x, \lambda)$ for $i=1,2$ be independent solutions of (4.2) which decay exponentially as $x \rightarrow-\infty$, and let $W_{i}^{+}(x, \lambda)$ for $i=1,2$ be such that $\mathbf{J}_{c} \overline{W_{i}^{+}}$are independent solutions of the adjoint of (4.2) which decay exponentially as $x \rightarrow+\infty$. For $\lambda \in \Lambda_{b}$, where $\Lambda_{b}$ is the subset of $\Lambda$ where individual vector-valued solutions are analytic, the symplectic Evans matrix is defined in [9] by

$$
\mathbf{E}_{b}(\lambda)=\left(\begin{array}{ll}
\Omega\left(W_{1}^{+}(x, \lambda), U_{1}^{-}(x, \lambda)\right) & \Omega\left(W_{1}^{+}(x, \lambda), U_{2}^{-}(x, \lambda)\right) \\
\Omega\left(W_{2}^{+}(x, \lambda), U_{1}^{-}(x, \lambda)\right) & \Omega\left(W_{2}^{+}(x, \lambda), U_{2}^{-}(x, \lambda)\right)
\end{array}\right)
$$

where $\Omega(\cdot, \cdot)$ is the symplectic form associated with the Hamiltonian system (2.16). The symplectic Evans function is then the determinant of this matrix. If there exists a $\lambda \in \Lambda_{b}$ with $D_{b}(\lambda)=\operatorname{det}\left(\mathbf{E}_{b}(\lambda)\right)=0$, then the basic solitary wave is linearly unstable. On the set $\Lambda_{b}, D_{b}(\lambda)$ and $D(\lambda)$ have the same zeros [9].

There is yet another form of the Evans function which uses individual vectors as in (6.2) but is analytic on the larger set $\Lambda$. This extension of the symplectic Evans matrix is introduced in Bridges and Derks [10]. It has the same form as (6.2) and the individual vectors in it span the same space as the vectors in (6.2) but they extend to analytic functions on the larger set $\Lambda$. Denote this Evans matrix by $\mathbf{E}(\lambda)$. In [10] it is proved that this matrix is analytic on $\Lambda$ and $\operatorname{det}(\mathbf{E}(\lambda))$ is equal to $D(\lambda)$ on $\Lambda$. Moreover, the sign of the first nonzero derivative of $\operatorname{det}\left(\mathbf{E}_{b}(\lambda)\right)$ is equal to the sign of the first nonzero derivative of $\operatorname{det}(\mathbf{E}(\lambda))$ at the origin.

In summary, the three forms of the Evans function can be used together to analyze the stability problem. The strategy here will be to show that $D(\lambda)=\operatorname{det}(\mathbf{E}(\lambda)) \rightarrow 1$ for $\lambda \rightarrow+\infty$ along the real axis. The geometry encoded in (4.2) will then be used to obtain explicit expressions for the derivatives of $D_{b}(\lambda)=\operatorname{det}\left(\mathbf{E}_{b}(\lambda)\right)$ at the origin following [9]. Then the equivalence between $D_{b}(\lambda)$ and $D(\lambda)$ in $\Lambda_{b}$ established in [10] is then used to prove a geometric instability condition. 
In section 8 , we will show that, for the region in parameter space associated with $C_{2} \beta>-2 c \beta$, an explicit geometric condition for the existence of at least one unstable eigenvalue can be deduced.

7. Large $\boldsymbol{\lambda}$ behavior of the Evans function. In this section, we will prove that $D(\lambda) \rightarrow 1$ as $\lambda \rightarrow+\infty$ along the real axis, for the Evans function associated with the Kawahara equation, linearized about a solitary wave. This will be proved by applying the Pego-Weinstein lemma in the appendix to the primary form of the Evans function (6.1) on wedge spaces.

The linear system has $n=6$ and $k=2$, hence the wedge space $\wedge^{2}\left(\mathbb{C}^{6}\right)$ has dimension $d=\left(\begin{array}{l}6 \\ 2\end{array}\right)=15$. Use the standard basis for $\mathbb{C}^{6}\left(\mathbf{e}_{1}=(1,0,0,0,0,0)^{T}\right.$, etc. $)$ and the standard lexically ordered induced basis, $\omega_{1}=\mathbf{e}_{1} \wedge \mathbf{e}_{2}, \ldots, \omega_{15}=\mathbf{e}_{5} \wedge \mathbf{e}_{6}$.

Let $\kappa=\lambda^{-1 / 5}$. For $\lambda$ large, the eigenvalues of the matrix $\mathbf{A}^{\infty}(\lambda)$ are

$$
0, B \kappa^{-1}+\mathcal{O}(\kappa), \frac{1}{4}(-1 \pm \sqrt{5}+\mathrm{i} \sqrt{2} \sqrt{5 \pm \sqrt{5}}) B \kappa^{-1}+\mathcal{O}(\kappa)
$$

where $B=\sqrt[5]{\frac{2}{-\beta}}$, and

$$
\frac{1}{4}(-1 \pm \sqrt{5}-\mathrm{i} \sqrt{2} \sqrt{5 \pm \sqrt{5}}) B \kappa^{-1}+\mathcal{O}(\kappa)
$$

Note that none of the eigenvalues is of order $\lambda$. This property corresponds to the fact that asymptotically $\mathbf{J}_{c}^{-1} \mathbf{M}$ is the main matrix in $\mathbf{A}^{\infty}(\lambda)$. And $\mathbf{J}_{c}^{-1} \mathbf{M}$ has only one eigenvalue -0 - and it has algebraic multiplicity 6 and geometric multiplicity 4 .

The eigenvalues of the induced matrix $\mathbf{A}_{\infty}^{(2)}(\lambda)$ in $\bigwedge^{(2)}\left(\mathbb{C}^{6}\right)$ are pairwise sums of eigenvalues of $\mathbf{A}^{\infty}(\lambda)$. Explicit expressions will not be given, but Figure 7.1 shows qualitatively the position of these eigenvalues relative to the eigenvalues of $\mathbf{A}^{\infty}(\lambda)$ in the complex $\mu$ plane.

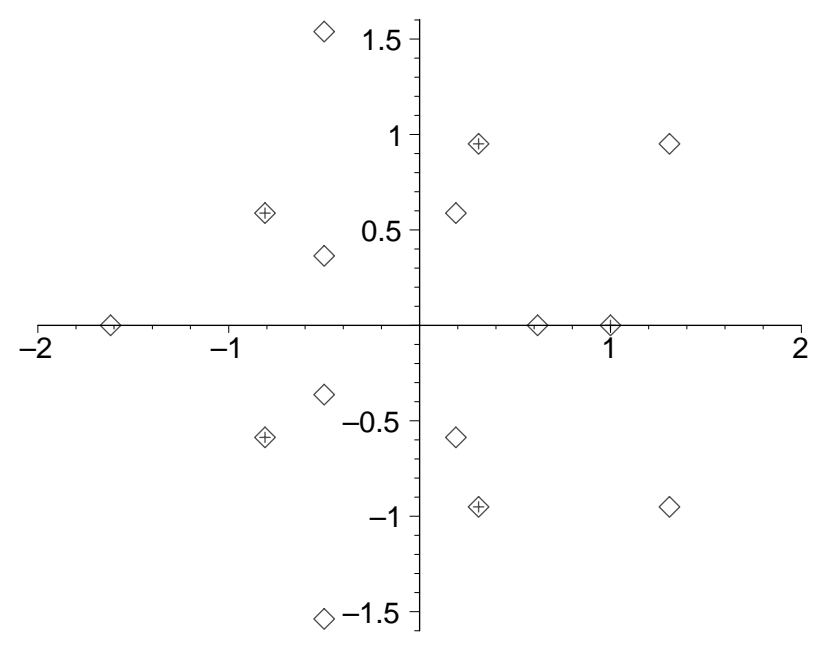

FIG. 7.1. For $B=1$ and $\lambda$ fixed (and large), the eigenvalues of the induced matrix $\mathbf{A}_{\infty}^{(2)}(\lambda)$ are denoted with a diamond and the eigenvalues of the matrix $\mathbf{A}^{\infty}(\lambda)$ with a cross (the zero eigenvalue of $\mathbf{A}^{\infty}(\lambda)$ is obscured by the axes). 
It is straightforward to verify that $\mathbf{A}_{\infty}^{(2)}(\lambda)$ is diagonalizable for $\lambda \in \Lambda$, using the explicit expressions for the eigenvectors. The eigenvector of $\mathbf{A}^{\infty}(\lambda)$ for the eigenvalue 0 is $\left(\kappa^{5}, 0,0,1,0,0\right)^{T}$ and the eigenvectors for a nonzero eigenvalue $\mu$ are

$$
\left(1, \mu, \mu^{2}, \mu c-\lambda,-\mu F_{1},-\beta \mu F_{2}\right)^{T},
$$

where $F_{1}$ and $F_{2}$ are defined in (4.5). The eigenvectors of the induced matrix are wedge products of pairs of those eigenvectors, so, using the above expressions, the matrix $\mathbf{V}(\lambda)$ in the Pego-Weinstein lemma of all eigenvectors can be formed.

The columns of $\mathbf{V}(\lambda)$, the eigenvectors of the induced matrix, have the following form:

$$
\left(\begin{array}{c}
\left(\nu_{0}-\mu_{0}\right) \kappa^{8} \\
\left(\nu_{0}^{2}-\mu_{0}^{2}\right) \kappa^{7} \\
c\left(\nu_{0}-\mu_{0}\right) \kappa^{8} \\
-\beta\left(\nu_{0}^{4}-\mu_{0}^{4}\right) \kappa^{5} \\
-\beta\left(\mu_{0}^{3}-\nu_{0}^{3}\right) \kappa^{6} \\
\mu_{0} \nu_{0}\left(\nu_{0}-\mu_{0}\right) \kappa^{6} \\
\left(\nu_{0}-\mu_{0}\right) \kappa^{3} \\
-\beta \mu_{0} \nu_{0}\left(\nu_{0}^{3}-\mu_{0}^{3}\right) \kappa^{4} \\
-\beta \mu_{0} \nu_{0}\left(\mu_{0}^{2}-\nu_{0}^{2}\right) \kappa^{5} \\
\left(\nu_{0}^{2}-\mu_{0}^{2}\right) \kappa^{2} \\
-\beta \mu_{0}^{2} \nu_{0}^{2}\left(\nu_{0}^{2}-\mu_{0}^{2}\right) \kappa^{3} \\
-\beta \mu_{0}^{2} \nu_{0}^{2}\left(\mu_{0}-\nu_{0}\right) \kappa^{4} \\
-\beta\left(\mu_{0}^{4}-\nu_{0}^{4}\right) \\
-\beta\left(\nu_{0}^{3}-\mu_{0}^{3}\right) \kappa \\
-\beta^{2} \mu_{0}^{3} \nu_{0}^{3}\left(\nu_{0}-\mu_{0}\right) \kappa^{2}
\end{array}\right)+\text { h.o.t. } \quad \text { or }\left(\begin{array}{c}
-\mu_{0} \kappa^{8} \\
-\mu_{0}^{2} \kappa^{7} \\
+2 \mu_{0} \kappa^{4} \\
\mu_{0}^{4} \beta \kappa^{5} \\
-\mu_{0}^{3} \beta \kappa^{6} \\
0 \\
\mu_{0} \kappa^{3} \\
0 \\
0 \\
\nu_{0} a r \\
\mu_{0}^{2} \kappa^{2} \\
0 \\
0 \\
\mu_{0}^{4} \beta \\
-\mu_{0}^{3} \beta \kappa \\
0
\end{array}\right)+\text { h.o.t., }
$$

where $\mu_{0}$ and $\nu_{0}$ are two different nonvanishing solutions of the first-order approximation of the eigenvalue $\mu$. Also, h.o.t. denotes the next order in each entry, except for the zero entries, which are identically zero.

Using this expression, we can now verify the three integral conditions in the Pego-Weinstein lemma. A straightforward but lengthy calculation gives

$$
\left\|\mathbf{V}(\lambda)^{-1}\left[\mathbf{A}^{(2)}(x, \lambda)-\mathbf{A}_{\infty}^{(2)}(\lambda)\right] \mathbf{V}(\lambda)\right\|=\mathcal{O}\left(\mathrm{e}^{-\delta|x|}\right), \quad \text { uniform in } \lambda
$$

for $\lambda$ and $|x|$ large, where $\delta$ represents the exponential decay rate of the basic solitary wave. Hence, the first two integral conditions are satisfied.

For the third condition, we have in general that

$$
\begin{aligned}
\left\|\mathbf{V}(\lambda)^{-1}\left[\mathbf{A}^{(2)}(x, \lambda)-\mathbf{A}_{\infty}^{(2)}(\lambda)\right] \zeta_{+}(\lambda)\right\| & =\left\|\mathbf{V}(\lambda)^{-1}\left[\mathbf{A}^{(2)}(x, \lambda)-\mathbf{A}_{\infty}^{(2)}(\lambda)\right] \mathbf{V}(\lambda) \mathbf{e}_{1}\right\| \\
& =\mathcal{O}\left(\mathrm{e}^{-\delta|x|}\right) .
\end{aligned}
$$

However, the integral of this function does not vanish for large $\lambda$, so the third integral condition of the Pego-Weinstein lemma will not be satisfied in general (for this we would require some decay rate in $\kappa / \lambda$ in the right-hand side).

However, under the main hypothesis needed in the applications presented herenamely $E_{r}(b, 0)=0$ - then $\mathbf{A}(x, \lambda)$ has a simpler structure and we get

$$
\left\|\mathbf{V}(\lambda)^{-1}\left[\mathbf{A}^{(2)}(x, \lambda)-\mathbf{A}_{\infty}^{(2)}(\lambda)\right] \zeta_{+}(\lambda)\right\|=\mathcal{O}\left(\mathrm{e}^{-\delta|x|} \kappa\right),
$$

which vanishes for large $\lambda$, and so the third integral condition is satisfied. In summary, we have proved the following. (This asymptotic result is actually true in a wedge about the real axis, but this property will not be needed here.) 
Proposition 7.1. Suppose $E_{r}(b, 0)=0$ and $C_{2} \beta+2 c \beta>0$, and let $D(\lambda)$ be the Evans function (6.1) linearized about a solitary wave of the form given in sections $2-3$. This function satisfies $D(\lambda) \rightarrow 1$ as $\lambda \rightarrow+\infty$ along the real axis.

8. A geometric instability criterion. Using the geometry of the symplectic Evans matrix, the slope of $\operatorname{det}\left(\mathbf{E}_{b}(\lambda)\right)$ for $\lambda$ small, real, and positive can be determined. Combining this result with the intermediate-value theorem and the large $\lambda$ asymptotics of $D(\lambda)$, the following geometric condition for linear instability is proved in $[9,10]$.

Define

$$
\chi_{00}^{-}=\left[\Omega\left(\Psi^{-}, D G_{\gamma}\left(Z_{0}^{-}\right)^{T} \Psi^{+}\right)\right]^{-1},
$$

and let $d_{\infty}$ be the value of the Evans function for some value of $\lambda \in \Lambda \cap \mathbb{R}$, usually $\lambda$ large. Then

$$
d_{\infty} \chi_{00}^{-}\left(\frac{\partial}{\partial c} I(\widetilde{Z})-\frac{1}{2} \omega\left(Z_{0}^{+}, \partial_{c} Z_{0}^{+}\right)\right)<0
$$

is a sufficient condition for linear instability of the solitary wave $\mathcal{G}_{a t+b x+\theta_{0}}(\widetilde{Z}(x-c t))$ (see [9], [10] for full details). It follows from section 7 that $d_{\infty}=+1$.

The expression (8.1) can be simplified by using the properties of the existing solitary wave, the special form of $\mathbf{M}$, and the fact that $D G_{\gamma}$ is the identity. By definition of the matrix $\mathbf{M}$, we have that

$$
I(\widetilde{Z})=-\int_{-\infty}^{\infty} \widetilde{q}_{2}\left(\widetilde{q}_{1}\right)_{x} d x+\left.\frac{1}{2} \widetilde{q}_{2} \widetilde{q}_{1}\right|_{-\infty} ^{\infty}
$$

Using that $\left(\widetilde{q}_{1}\right)_{x}=\widetilde{q}_{2}-b$ and $\widetilde{q}_{2}(-\infty)=b=\widetilde{q}_{2}(\infty)$, this implies that

$$
I(\widetilde{Z})=-\int_{-\infty}^{\infty}\left(\widetilde{q}_{2}-b\right)\left(\widetilde{q}_{2}-\frac{b}{2}\right) d x .
$$

Also,

$$
\frac{1}{2} \omega\left(Z_{0}^{+}, \partial_{c} Z_{0}^{+}\right)=-\frac{b}{2} \frac{\partial}{\partial c} \int_{-\infty}^{\infty}\left(\widetilde{q}_{2}-b\right) d x
$$

hence

$$
\frac{\partial}{\partial c} I(\widetilde{Z})-\frac{1}{2} \omega\left(Z_{0}^{+}, \partial_{c} Z_{0}^{+}\right)=-\frac{\partial}{\partial c} \int_{-\infty}^{\infty}\left(\widetilde{q}_{2}-b\right)^{2} d x
$$

Finally, since $\Psi^{-}$is an eigenvector at $\lambda=0$ with eigenvalue $\delta$ and $\Psi^{+}$is an eigenvector at $\lambda=0$ with eigenvalue $-\delta$, there are constants $C_{5}^{ \pm}$such that $\Psi^{ \pm}=$ $C_{5}^{ \pm} U_{\text {ev }}(\mp \delta, 0)$ with $U_{\text {ev }}(\mu, \lambda)$ given by (4.5). Hence

$$
\Psi^{-}=\frac{C_{5}^{-}}{C_{5}^{+}}\left(\Psi^{+}+2 \delta C_{5}^{+}\left(0,1,0, c,-F_{q r}(b, 0),-E_{q}(b, 0)+\beta \delta^{2}\right)\right)
$$

and

$$
\left(\chi_{00}^{-}\right)^{-1}=-2 \delta^{3} C_{5}^{-} C_{5}^{+}\left[\alpha+F_{r r}(b, 0)-2 E_{q}(b, 0)+2 \beta \delta^{2}\right]=-2 \delta^{3} C_{5}^{-} C_{5}^{+}\left[C_{1}+2 \beta \delta^{2}\right] .
$$


Since $\delta$ is the positive real eigenvalue, (4.6) gives that

$$
C_{1}+2 \beta \delta^{2}=C_{1}+\sqrt{C_{1}^{2}+4 \beta\left(C_{2}+2 c\right)}-C_{1}=\sqrt{C_{1}^{2}+4 \beta\left(C_{2}+2 c\right)}>0 .
$$

Also $2 \delta^{3}>0$, so we can conclude the following.

Theorem 8.1. Define $C_{5}^{ \pm}=\lim _{x \rightarrow \pm \infty} e^{ \pm \delta x}\left(\widetilde{q}_{2}(x)-b\right)$. If $E=0, F_{q q}(b, 0) \beta>$ $-2 c \beta$, and

$$
C_{5}^{-} C_{5}^{+} \frac{\partial}{\partial c} \int_{-\infty}^{\infty}\left(\widetilde{q}_{2}(x)-b\right)^{2} d x<0
$$

then the solitary wave solution $\widetilde{Z}(x ; a, b, c)$ of the generalized Kawahara equation (1.1) is unstable.

If $b=0$ and $\widetilde{q}_{2}$ is even, then $C_{5}^{-} C_{5}^{+}>0$. In this case the condition for instability is reminiscent of the abstract condition deduced from the energy-momentum method in Grillakis, Shatah, and Strauss [18] and Bona, Souganidis, and Strauss [4], although here there is no requirement on the second variation of the constrained critical point problem. If $b \neq 0$ and $\left(\widetilde{q}_{2}-b\right)$ is not even - and it is known from numerical results that such solutions exist [11] - then $C_{5}^{-} C_{5}^{+}$may be negative, in which case the condition for instability is precisely opposite that of the energy-momentum characterization (see section 5 of [7] for an example where this switch can occur).

In the next two sections we will apply this theorem to two known classes of solitary wave states of the generalized Kawahara equation.

9. Example: Kichenasammy-Olver nonlinearity. In this section we consider a class of generalized Kawahara equations, as considered in Kichenassamy and Olver [25], i.e., $E=0$ and $F(q, r)=-A q r^{2}+\frac{1}{2} c_{1} q^{2}+\frac{1}{3} c_{2} q^{3}+\frac{1}{4} c_{3} q^{4}$. This implies that

$$
f\left(u, u_{x}, u_{x x}\right)=A\left(u_{x}\right)^{2}+2 A u u_{x x}+c_{1} u+c_{2} u^{2}+c_{3} u^{3} .
$$

In [25], it is shown that if

$$
c_{2}=\frac{3 \alpha A}{5 \beta} \quad \text { and } \quad c_{3}=-\frac{2 A^{2}}{5 \beta},
$$

then the Kawahara equation with $f$ given by (9.1) has a two-parameter family of exact solitary wave solutions of the form

$$
\widetilde{q}_{2}(x)=u(x)=-\frac{10 \beta \phi^{2}}{A} \operatorname{sech}^{2}(\phi(x-c t))+b,
$$

where $\phi$ is a positive solution of

$$
80\left(\beta \phi^{2}\right)^{2}-20 \beta \phi^{2}(2 b A-\alpha)+6 b A(b A-\alpha)-5 \beta\left(c_{1}+2 c\right)=0,
$$

which is equivalent to the condition $\Delta( \pm 2 \phi, 0)=0$. This condition implies that if $\beta(2 b A-\alpha) \geq 0$, then the family exists for $40 c \beta>4 b^{2} A^{2}-4 b A \alpha-5 \alpha^{2}-20 c_{1} \beta$. If $\beta(2 b A-\alpha)<0$, then the family exists for $40 c \beta>24 b^{2} A^{2}-24 b A \alpha-20 c_{1} \beta$.

In terms of the notation of section 3 , we have $\delta=2 \phi$ and

$$
\Psi^{ \pm}=\frac{40 \beta \phi^{2}}{A}\left(-1, \pm 2 \phi,-4 \phi^{2}, \pm 2 c \phi,-4 \phi^{2}\left(-4 \beta \phi^{2}-\alpha+2 b A\right), \pm 8 \beta \phi^{3}\right),
$$


i.e., $C_{5}^{ \pm}=-\frac{40 \beta \phi^{2}}{A}$. A tedious but straightforward calculation demonstrates that $\lim _{x \rightarrow \pm \infty} \partial_{x}\left[e^{ \pm \delta x} \widetilde{Z}_{x}\right]=0$. So all the conditions of section 3 are satisfied.

Next we look at section 4 . For this example we have

$$
C_{1}=\alpha-2 A b \quad \text { and } \quad C_{2}=c_{1}+\frac{6 A b}{5 \beta}(\alpha-A b) .
$$

So an eigenvector can be written as

$$
U_{\mathrm{ev}}(\mu, \lambda)=\left(1, \mu, \mu^{2},-\lambda+\mu c,-\mu^{2}\left(\alpha-2 A b+\beta \mu^{2}\right), \beta \mu^{3}\right) .
$$

In order to have $C_{2} \beta>-2 c \beta$, the analysis will be restricted to the case

$$
40 c \beta>-20 \beta c_{1}-24 A b(\alpha-A b),
$$

which is exactly the sufficient condition for the existence of the family of solitary waves. This condition is also necessary if $\beta(2 b A-\alpha)<0$.

Now we are ready to apply the instability criterion. A straightforward calculation shows that

$-\int_{-\infty}^{\infty}(u(x)-b)^{2} d x=-\frac{400 \phi^{3} \beta^{2}}{3 A^{2}}, \quad$ hence $-\frac{\partial}{\partial c} \int_{-\infty}^{\infty}(u(x)-b)^{2} d x=-\frac{400 \phi^{2} \beta^{2}}{A^{2}} \frac{\partial}{\partial c} \phi$.

To determine $\frac{\partial}{\partial c} \phi$, we differentiate (9.2). This gives

$$
\frac{\partial}{\partial c} \phi=\frac{1}{4 \phi\left(8 \beta \phi^{2}-2 b A+\alpha\right)} .
$$

Also,

$$
\left(\chi_{00}^{-}\right)^{-1}=\left(\mathbf{J}_{c} \Psi^{-}, \Psi^{+}\right)=-\frac{25600 \beta^{2} \phi^{7}}{A^{2}}\left(8 \beta \phi^{2}+\alpha-2 b A\right) .
$$

So

$$
\chi_{00}^{-}\left(\frac{\partial}{\partial c} I(\widetilde{Z})-\frac{1}{2} \omega\left(Z_{0}^{+}, \partial_{c} Z_{0}^{+}\right)\right)=\frac{A^{2}}{25600 \beta^{2} \phi^{7}} \frac{1}{4 \phi} \frac{400 \phi^{2} \beta^{2}}{A^{2}}=\frac{1}{256 \phi^{6}}>0 .
$$

Since the sufficient geometric condition for linear instability is not met, this suggests that the solitary wave is "stable." However, there could still be unstable $\lambda$-eigenvalues on the positive real $\lambda$-axis, but there would be two or more. The solitary wave could also be unstable due to unstable eigenvalues with nonzero imaginary part.

10. Example: Levandosky's homogeneous nonlinearity. In this section we consider a class of nonlinearities for (1.1) which includes the equations considered by Levandosky [26]. We take $E=0$ and $F$ homogeneous of degree $p+1$ for some $p>1$, i.e.,

$$
F(\nu q, \nu r)=\nu^{p+1} F(q, r) \quad \text { for all } \nu \geq 0 \text { and }(q, r) \in \mathbb{R}^{2} .
$$

In [26], it is proved that the following condition is sufficient for the existence of a one-parameter family of solitary waves,

$$
\int_{-\infty}^{\infty} F\left(u, u_{x}\right) d x>0 \quad \text { for some } u \in H^{2}(\mathbb{R}), b=0, \beta=1 \text {, and } 4 c>(\max \{\alpha, 0\})^{2} .
$$


Recall $\mathcal{I}(u)=\frac{1}{2} \int_{-\infty}^{\infty}|u(x)|^{2} d x$ and assume that a family of solitary waves $\widehat{u}(x ; c)$ exists, where $\widehat{u}$ travels with speed $c$. In [26] it is also shown that under the above conditions, the solitary waves are stable if $\frac{d}{d c} \mathcal{I}(\widetilde{u})>0$ and unstable if $\frac{d}{d c} \mathcal{I}(\widetilde{u})<0$ and $p \geq 2$. When $b=0$ in the formulation in sections $2-6$, this instability condition agrees with Theorem 8.1 if $C_{5}^{+} C_{5}^{-}>0$.

First, we embed the solitary wave state in a two-parameter family, allowing for $b$ to be nonzero. Then we show how our theory recovers the result in [26], without any specific use of the variational principle associated with the energy-momentum characterization. For definiteness, consider the case $\alpha=0$ and take $F$ to be homogeneous in $r$ itself, i.e., there is some $0 \leq n \leq p+1$ such that

$$
F(q, \nu r)=\nu^{n} F(q, r) \quad \text { for all } \nu \geq 0 \text { and }(q, r) \in \mathbb{R}^{2} .
$$

This homogeneity condition in $r$ implies that $F_{q q}(b, 0)=0$, hence the condition on $c$ of Theorem 8.1 becomes $c>0$.

When there exists a family of solitary wave solutions $\{\widetilde{Z}(x ; a, b, 1) \mid a \in \mathbb{R}, b \in \mathbb{R}\}$ with speed $c=1$, then we can construct a family of solitary wave solutions with speed $c>0$ :

$$
\widetilde{Z}(x ; a, b, c)=c^{\frac{4-n}{4(p-1)}} \operatorname{diag}\left(c^{-\frac{1}{4}}, 1, c^{\frac{1}{4}}, c, c^{\frac{3}{4}}, c^{\frac{1}{2}}\right) \widetilde{Z}\left(c^{\frac{1}{4}} x ; a c^{\frac{n-4 p}{4(p-1)}}, b c^{-\frac{4-n}{4(p-1)}}, 1\right) .
$$

Indeed, $\widetilde{Z}(x ; a, b, c)$ as defined above satisfies

$$
\begin{aligned}
\mathbf{J}_{c} D_{x} \widetilde{Z}(x ; a, b, c)= & c^{\frac{1}{4}} c^{\frac{4-n}{4(p-1)}} \mathbf{J}_{c} \operatorname{diag}\left(c^{-\frac{1}{4}}, 1, c^{\frac{1}{4}}, c, c^{\frac{3}{4}}, c^{\frac{1}{2}}\right) \mathbf{J}_{1}^{-1} \mathbf{J}_{1} \\
& \widetilde{Z}_{x}\left(c^{\frac{1}{4}} x ; a c^{\frac{n-4 p}{4(p-1)}}, b c^{-\frac{4-n}{4(p-1)}}, 1\right) \\
= & c^{\frac{1}{4}} \mathbf{J}_{c} \operatorname{diag}\left(c^{-\frac{1}{4}}, 1, c^{\frac{1}{4}}, c, c^{\frac{3}{4}}, c^{\frac{1}{2}}\right) \mathbf{J}_{1}^{-1} \\
& \quad\left(\nabla S(\widetilde{Z})-a c^{\frac{n-4 p}{4(p-1)}} \nabla P(\widetilde{Z})-b c^{-\frac{4-n}{4(p-1)}} \nabla Q(\widetilde{Z})\right) \\
= & \nabla S(\widetilde{Z}(x ; a, b, c))-a \nabla P(\widetilde{Z}(x ; a, b, c))-b \nabla Q(\widetilde{Z}(x ; a, b, c)) .
\end{aligned}
$$

This implies that for $b=0$ or $n=4$

$$
\begin{aligned}
\int_{-\infty}^{\infty}\left(\widetilde{q}_{2}(x ; a, b, c)-b\right)^{2} d x & =c^{\frac{4-n}{2(p-1)}} \int_{-\infty}^{\infty}\left(\widetilde{q}_{2}\left(c^{\frac{1}{4}} x ; a c^{\frac{n-4 p}{4(p-1)}}, b c^{-\frac{4-n}{4(p-1)}}, 1\right)\right. \\
& \left.-b c^{-\frac{4-n}{4(p-1)}}\right)^{2} d x \\
= & c^{\frac{4-n}{2(p-1)}} c^{-\frac{1}{4}} \int_{-\infty}^{\infty}\left(\widetilde{q}_{2}\left(s ; a c^{\frac{n-4 p}{4(p-1)}}, b c^{-\frac{4-n}{4(p-1)}}, 1\right)\right. \\
& \left.-b c^{-\frac{4-n}{4(p-1)}}\right)^{2} d s \\
= & c^{\frac{4-n}{2(p-1)}-\frac{1}{4}} \int_{-\infty}^{\infty}\left(\widetilde{q}_{2}\left(s ; a c^{\frac{n-4 p}{4(p-1)}}, b, 1\right)-b\right)^{2} d s .
\end{aligned}
$$

In the last step we used that $b=0$ or $n=4$, hence $b c^{-\frac{4-n}{4(p-1)}}=b$. Since the defining equation for $\widetilde{q}_{2}$ does not depend on $a$, this integral will not depend on $a$ either and we can put $a=0$; hence,

$$
\int_{-\infty}^{\infty}\left(\widetilde{q}_{2}(x ; a, b, c)-b\right)^{2} d x=c^{\frac{9-2 n-p}{4(p-1)}} \int_{-\infty}^{\infty}\left(\widetilde{q}_{2}(s ; 0, b, 1)-b\right)^{2} d s .
$$


So

$$
\frac{\partial}{\partial c} \int_{-\infty}^{\infty}\left(\widetilde{q}_{2}(x ; a, b, c)-b\right)^{2} d x=\frac{9-2 n-p}{4(p-1)} c^{\frac{13-2 n-5 p}{4(p-1)}} \int_{-\infty}^{\infty}\left(\widetilde{q}_{2}(s ; 0, b, 1)-b\right)^{2} d s .
$$

Furthermore,

$$
C_{5}^{+}(a, b, c) C_{5}^{-}(a, b, c)=c^{\frac{4-n}{2(p-1)}} C_{5}^{+}\left(a c^{\frac{n-4 p}{4(p-1)}}, b, 1\right) C_{5}^{-}\left(a c^{\frac{n-4 p}{4(p-1)}}, b, 1\right) .
$$

It is difficult to verify the hypothesis (3.2) for this example and therefore we assume (3.2) is satisfied. Then with

$$
C_{5}^{+}\left(a c^{\frac{n-4 p}{4(p-1)}}, b, 1\right) C_{5}^{-}\left(a c^{\frac{n-4 p}{4(p-1)}}, b, 1\right) \int_{-\infty}^{\infty} \widetilde{q}_{2}^{2}(s ; 0, b, 1) d s>0 .
$$

Theorem 8.1 shows that the solitary waves are unstable if $9-2 n-p<0$, i.e., $p>9-2 n$. These instability results agree with those in [26] when an error in Lemma 3.3 in [26] is corrected. (The $(p+1)(4-\beta)$ in the numerator of the expression for $\gamma$ should be replaced by $4(p+1)-2 \beta$.

If $n=4$, then $b \neq 0$ is allowed and we obtain that the wave is always unstable since

$$
\frac{\partial}{\partial c} \int_{-\infty}^{\infty}\left(\widetilde{q}_{2}(x ; a, b, c)-b\right)^{2} d x=-\frac{1}{4} c^{-\frac{5}{4}} \int_{-\infty}^{\infty} \widetilde{q}_{2}^{2}(s ; 0, b, 1) d s<0 .
$$

Appendix. Large $\boldsymbol{\lambda}$ behavior and the Pego-Weinstein lemma. The following result is a generalization of Proposition 1.17 in Pego and Weinstein [29], which gives a sufficient condition for $D(\lambda) \rightarrow 1$ as $\lambda \rightarrow+\infty$ along the real $\lambda$-axis. Consider the system

$$
\mathbf{u}_{x}=\mathbf{A}(x, \lambda) \mathbf{u}, \quad \mathbf{u} \in \mathbb{C}^{n}, \quad \lambda \in \Lambda,
$$

where $\Lambda$ is an open simply connected subset of $\mathbb{C}$, and $\Lambda$ includes a wedge about the real axis in which we can take $|\lambda| \rightarrow \infty$. The spectrum of $\mathbf{A}^{\infty}(\lambda)$, where

$$
\mathbf{A}^{\infty}(\lambda)=\lim _{|x| \rightarrow \infty} \mathbf{A}(x, \lambda)
$$

is assumed to have $k$-eigenvalues with negative real part and $n-k$ with nonnegative real part. A critical hypothesis in Proposition 1.17 in [29] is that $k=1$. However, this hypothesis is not essential if we take into account that on $\bigwedge^{k}\left(\mathbb{C}^{n}\right)$ the induced matrix

$$
\mathbf{A}_{\infty}^{(k)}(\lambda) \stackrel{\text { def }}{=} \bigwedge^{k}\left(\mathbf{A}^{\infty}(\lambda)\right)
$$

has a unique simple eigenvalue of largest negative real part. Then working on $\bigwedge^{k}\left(\mathbb{C}^{n}\right)$ with the Evans function also on the exterior algebra, the proof of Proposition 1.17 carries over [10]. The precise statement of the result needed in this paper is given below. Although stated in a substantially more general form, the proof given in [29] carries over almost verbatim.

Pego-Weinstein lemma. Consider the system (A.1)-(A.2) and suppose that for all $\lambda \in \Lambda$ the eigenvalue of $\mathbf{A}_{\infty}^{(k)}(\lambda)$ with largest negative real part is unique and simple. Denote this eigenvalue by $\alpha(\lambda)$ and its (analytic choice of) right eigenvector by $\zeta(\lambda)$ 
and its (analytic choice of) left eigenvector by $\eta(\lambda)$ with normalization $\llbracket \eta, \zeta \rrbracket_{k}=1$, where $\llbracket \cdot, \cdot \rrbracket_{k}$ is the induced inner product on $\Lambda^{k}\left(\mathbb{C}^{n}\right)$.

Let $\mathbf{U}(x, \lambda) \in \bigwedge^{k}\left(\mathbb{C}^{n}\right)$ be the solution of the system

$$
\mathbf{U}_{x}=\mathbf{A}^{(k)}(x, \lambda) \mathbf{U} \quad \text { satisfying } \quad \lim _{x \rightarrow+\infty} \mathrm{e}^{-\alpha(\lambda) x} \mathbf{U}(x, \lambda)=\zeta(\lambda) \in \bigwedge^{k}\left(\mathbb{C}^{n}\right) .
$$

Similarly, let $\mathbf{W}(x, \lambda) \in \bigwedge^{k}\left(\mathbb{C}^{n}\right)$ be the solution of the system

$$
\mathbf{W}_{x}=-\mathbf{A}^{(k)}(x, \lambda)^{T} \mathbf{W} \quad \text { satisfying } \quad \lim _{x \rightarrow-\infty} \mathrm{e}^{\alpha(\lambda) x} \mathbf{W}(x, \lambda)=\eta(\lambda) \in \bigwedge^{k}\left(\mathbb{C}^{n}\right) .
$$

In terms of these functions, the Evans function (6.1) can be expressed in the form

$$
D(\lambda)=\mathbf{W}(0, \lambda) \cdot \mathbf{U}(0, \lambda) \stackrel{\text { def }}{=}\langle\overline{\mathbf{W}(0, \lambda)}, \mathbf{U}(0, \lambda)\rangle,
$$

where $\langle\cdot, \cdot\rangle$ is a standard Hermitian inner product on $\mathbb{C}^{d}$ and $d=\operatorname{dim} \bigwedge^{k}\left(\mathbb{C}^{n}\right)$.

Now, suppose $\mathbf{A}_{\infty}^{(k)}(\lambda)$ is diagonalizable for large $\lambda$ and let $\mathbf{V}(\lambda) \in \mathbb{C}^{d \times d}$ be the matrix of right eigenvectors such that the first column is $\zeta(\lambda)$. If

$$
\begin{aligned}
& \int_{-\infty}^{+\infty}\left\|\mathbf{V}(\lambda)^{-1}\left[\mathbf{A}^{(k)}(x, \lambda)-\mathbf{A}_{\infty}^{(k)}(\lambda)\right] \mathbf{V}(\lambda)\right\| \mathrm{d} x \leq C, \quad \text { independent of } \lambda, \\
& \int_{|x| \geq x_{0}}\left\|\mathbf{V}(\lambda)^{-1}\left[\mathbf{A}^{(k)}(x, \lambda)-\mathbf{A}_{\infty}^{(k)}(\lambda)\right] \mathbf{V}(\lambda)\right\| \mathrm{d} x \rightarrow 0, \quad \text { as } x_{0} \rightarrow \infty, \text { uniformly in } \lambda, \\
& \int_{-\infty}^{+\infty}\left\|\mathbf{V}(\lambda)^{-1}\left[\mathbf{A}^{(k)}(x, \lambda)-\mathbf{A}_{\infty}^{(k)}(\lambda)\right] \zeta(\lambda)\right\| \mathrm{d} x \rightarrow 0, \quad \text { as }|\lambda| \rightarrow \infty,
\end{aligned}
$$

then

$$
\mathbf{V}(\lambda)^{-1} \mathbf{U}(0, \lambda)=\mathbf{V}(\lambda)^{-1} \zeta(\lambda)+o(1) \quad \text { for }|\lambda| \rightarrow \infty
$$

and $\mathbf{W}(0, \lambda) \mathbf{V}(\lambda)$ is bounded with

$$
\mathbf{W}(0, \lambda) \mathbf{V}(\lambda) \mathbf{e}_{1}=\mathbf{W}(0, \lambda) \zeta(\lambda)=1+o(1) \quad \text { for }|\lambda| \rightarrow \infty .
$$

The two results (A.3) and (A.4) imply that $D(\lambda) \rightarrow 1$ as $|\lambda| \rightarrow \infty$.

\section{REFERENCES}

[1] J. Alexander, R. Gardner, and C.K.R.T. Jones, A topological invariant arising in the stability analysis of traveling waves, J. Reine Angew. Math., 410 (1990), pp. 167-212.

[2] T.B. Benjamin, The stability of solitary waves, Proc. Roy. Soc. (London) Ser. A, 328 (1972), pp. $153-183$.

[3] J.L. Bona, On the stability of solitary waves, Proc. Roy. Soc. (London) Ser. A, 344 (1975), pp. 363-374.

[4] J.L. Bona, P.E. Souganidis, And W.A. Strauss, Stability and instability of solitary waves of KdV type, Proc. Roy. Soc. (London) Ser. A, 411 (1987), pp. 395-411.

[5] T.J. BRIDGeS, Multi-symplectic structures and wave propagation, Math. Proc. Cambridge Philos. Soc., 121 (1997), pp. 147-190.

[6] T.J. BRIDGes, Toral equivariant partial differential equations and quasiperiodic patterns, Nonlinearity, 11 (1998), pp. 467-500.

[7] T.J. Bridges AND G. Derks, Unstable eigenvalues, and the linearization about solitary waves and fronts with symmetry, Proc. Roy. Soc. (London) Ser. A, 455 (1999), pp. 2427-2469.

[8] T.J. Bridges and G. Derks, Hodge duality and the Evans function, Phys. Lett. A, 251 (1999), pp. 363-372. 
[9] T.J. Bridges And G. Derks, The symplectic Evans matrix, and the instability of solitary waves and fronts with symmetry, Arch. Ration. Mech. Anal., 156 (2001), pp. 1-87.

[10] T.J. Bridges and G. Derks, Constructing the Symplectic Evans Matrix Using Maximally Analytic Individual Vectors, preprint, University of Surrey, Surrey, UK, 2001; also available online from http://www.maths.surrey.ac.uk/personal/st/T.Bridges/PAPERS/SEMpaper.ps.

[11] A.R. ChampNeys, Homoclinic orbits in reversible systems and their applications in mechanics, fluids and optics, Phys. D, 112 (1998), pp. 158-186.

[12] A.R. Champneys And M.D. Groves, A global investigation of solitary-wave solutions to a two-parameter model for water waves, J. Fluid Mech., 342 (1997), pp. 199-229.

[13] W. Craig and M.D. Groves Hamiltonian long-wave approximations to the water-wave problem, Wave Motion, 19 (1994), pp. 367-389.

[14] X. DAI AND J. DAI, Some solitary wave solutions for families of generalized higher-order KdV equations, Phys. Lett. A, 142 (1989), pp. 367-370.

[15] B. Dey, A. Khare, And C.N. Kumar, Stationary solutions of the fifth-order KdV-type equations and their stabilization, Phys. Lett. A, 223 (1996), pp. 449-452.

[16] F. Dias And E.A. Kuznetsov, Nonlinear stability of solitons in the fifth-order Korteweg-de Vries equation, Phys. Lett. A, 263 (1999), pp. 98-104.

[17] J. W. Evans, Nerve axon equations IV. The stable and unstable impulse, Indiana Univ. Math. J., 24 (1975), pp. 1169-1190.

[18] M. Grillakis, J. Shatah, and W. Strauss, Stability theory of solitary waves in the presence of symmetry, I, J. Funct. Anal., 74 (1987), pp. 160-197.

[19] M. Groves, Solitary-wave solutions to a class of fifth-order model equations, Nonlinearity, 11 (1998), pp. 341-353.

[20] G. Huang, S. Luo, And X. DAI, Exact and explicit solitary-wave solutions to a model equation for water waves, Phys. Lett. A, 139 (1989), pp. 373-374.

[21] A.T. Ill'ichev And A.Y. Semenov, Stability of solitary waves in dispersive media described by a fifth-order evolution equation, Theor. Comput. Fluid Dyn., 3 (1992), pp. 307-326.

[22] B.B. Kadomtsev and V.I. Petviashvili, On the stability of solitary waves in weakly dispersing media, Sov. Phys. Dokl., 15 (1970), pp. 539-541.

[23] V.I. Karpman, Stabilization of soliton instabilities by higher-order dispersion: KdV-type equations, Phys. Lett. A, 210 (1996), pp. 77-84.

[24] R. Kawahara, Oscillatory solitary waves in dispersive media, J. Phys. Soc. Japan, 33 (1972), pp. 260-264.

[25] S. Kichenassamy and P.J. Olver, Existence and nonexistence of solitary wave solutions to higher-order model evolution equations, SIAM J. Math. Anal., 23 (1992), pp. 1141-1166.

[26] S.P. Levandosky, A stability analysis for fifth-order water-wave models, Phys. D, 125 (1999), pp. 222-240.

[27] M.S. Longuet-Higgins, On the mass, momentum, energy and circulation of a solitary wave, Proc. Roy. Soc. (London) Ser. A, 337 (1974), pp. 1-37.

[28] P.J. Olver, Hamiltonian perturbation theory and water waves, Contemp. Math., 28 (1984), pp. 231-249.

[29] R.L. Pego and M.I. Weinstein, Eigenvalues, and instabilities of solitary waves, Philos. Trans. Roy. Soc. London Ser. A, 340 (1992), pp. 47-94. 